\title{
Resonance features of rock slope with anti-dip weak interlayer under seismic actions
}

\author{
Tong Wang ${ }^{1} \cdot$ Jingli Huang ${ }^{1} \cdot$ Qiuling Lang ${ }^{1} \cdot$ Lisha Liu $^{1}$ \\ Received: 13 November 2020 / Accepted: 23 January 2021 / Published online: 15 February 2021 \\ (C) The Author(s) 2021
}

\begin{abstract}
Resonance avoidance is an important topic in engineering seismic design. However, there is little report on the resonance features of rock slopes with anti-dip weak rock layers under seismic actions. Through finite-element method, this paper carries out modal analysis and harmonic response analysis on a slope, captures the natural frequencies and vibration modes of the slope, and plots the resonance curves about displacement frequency relationships. The results show that slope resonance is greatly affected by damping ratio: the greater the damping ratio, the lower the resonance peak; the inverse is also true. This means that weak and broken rock mass is capable of absorbing shocks, but not necessarily easy to be damaged. On the contrary, broken rock mass has poor mechanical performance and is prone to damage under small vibration. Besides, the resonance effect of the slope is mainly excited by the natural frequencies of the first three orders; the vertical resonance displacement peaked at about $1.1 \mathrm{~Hz}$, while the horizontal resonance displacement peaked at about $1.38 \mathrm{~Hz}$. From the vibration modes and frequency response curves, the resonance peak of the slope is amplified in the vertical direction and on the free face, and the maximum resonance displacement appears on the surface of the slope, indicating that the strongest resonance occurs on slope surface. This research fully clarifies the stability and dynamic response law of rock slopes with anti-dip weak interlayer under seismic actions, laying a solid basis for the mic fortification, landslide prediction, and landslide control of slopes.
\end{abstract}

Keywords Rock slope $\cdot$ Weak interlayer $\cdot$ Resonance features $\cdot$ Seismic actions $\cdot$ Finite-element method

\section{Introduction}

The West Pit mine is located in the southeast of Fushun, a mining city in northeastern China. After being excavated for over a century, the mine has grown into the largest pit in Asia, with a length of $6.6 \mathrm{~km}$ from east to west, a width of $2.2 \mathrm{~km}$ from north to south, and a depth of about $400-500 \mathrm{~m}$. The total volume of the pit reaches 1.7 billion $\mathrm{km}^{3}$. The north side of the mine is on the edge of downtown Fushun. If a landslide occurs on the northern slope, it will cause heavy casualties and property losses. All the nearby industrial and civil facilities

Responsible Editor: Ahmed Farouk

This article is part of the Topical Collection on Big Data and Intelligent Computing Techniques in Geosciences

Tong Wang

wt09143323@163.com

1 School of Prospecting \& Surveying Engineering, Changchun Institute of Technology, Changchun 130061, China will be seriously affected, and the safety of the entire city will be put into jeopardy.

The northern slope of the West Pit mine lies to the east of Tanlu fault zone. As the largest seismic belt in China, Tanlu fault zone is a long-term active tectonic seismic belt with frequent earthquakes. Despite the absence of destructive earthquakes, Fushun has been frequently hit by small earthquakes. In the wake of the Haicheng earthquake, a total of 36 small earthquakes occurred successively in Fushun, more frequent than any other period in history.

The Haicheng earthquake is related to the new tectonic activities of the Tanlu fault zone. The seismic waves propagated along the Huhe fault to Fushun, reaching the intensity of VI. In other words, after the main shock of the Haicheng earthquake, the residual strain energy was transmitted to Fushun via the Tanlu fault zone.

According to the seismic data in China and abroad, the tectonic belts that have been struck with large earthquakes are very likely to suffer from such earthquakes again. Thus, it is impossible to rule out the possibility of large tectonic earthquakes in Fushun, which resides on the Hunhe fault zone. 
Therefore, this paper investigates the northern slope of the West Pit mine from the seismic perspective, and discusses the dynamic stability and dynamic response of that slope through static calculation.

Many scholars have studied the features and change laws of the dynamic response of slopes under dynamic loads (Zhou 2010; Zheng 2000; Gao et al. 2019; Cardarilli et al. 2019; Du et al. 2001; Qiu et al. 2018). Both engineers and rock dynamics researchers are eagerly waiting for effectively methods to analyze and control slope stability (Luo et al. 2020; Onyango and Zhang 2019; Li and Cai 2020). Shuren et al. (1999) measured the aftershocks of the San Fernando earthquake and found that the seismic acceleration at the top of the slope is many times larger than that at the slope foot. With the aid of Fast Lagrangian Analysis of Continua in 3 Dimensions (FLAC3D), Xu (2006) established a numerical analysis model for slope dynamics, probed deep into the dynamic response features of a slope, and drew the following conclusions: In the vertical and horizontal directions, the slope significantly amplified the input seismic wave; the low- and high-frequency components of the seismic wave were amplified and filtered by the rock-soil mass, respectively; the acceleration amplification factor of the input seismic wave was negatively correlated with the amplitude and frequency, and not greatly affected by the duration of the seismic wave; on the same vertical plane, the acceleration amplification factor increased with the elevation, and the increasing rate peaked on the top of the slope; and at the same elevation, the acceleration magnification factor increased from the interior to the surface of the slope.

Zhang (2008) discovered the following through blasting vibration tests: The dynamic seismic response of the slope depends heavily on the frequency of the input seismic wave and became significant as that frequency approached the superior frequency of the slope; the duration of the input seismic wave had an insignificant effect on the amplification effect. Yang (2007) adopted ANSYS, a finite-element analysis software, to analyze the dynamic seismic response of a homogeneous slope, loaded the Tianjin seismic wave onto the model for Wilson-theta time history analysis, and examined the dynamic response of the slope in terms of displacement, stress, velocity, and acceleration. The simulation results were post-processed by the MohrCoulomb theory, producing the cloud map on the safety factor of the slope. The analysis shows that the most significant seismic response of the slope manifested in acceleration, followed in turn by velocity and displacement; the strongest fluctuations of displacement, velocity, and acceleration were observed on the top of the slope, and the second strongest fluctuations were observed at the foot of the slope; the maximum and minimum of the safety factor appeared at the slope foot and on the slope top, respectively; the part of the potential sliding surface passing through the free face tended to slip.

Taking the high slope of the Mianmao Highway in Sichuan, China, as the prototype, Zhang (1998) coupled finite-element method with infinite-element method to analyze the influence of geotechnical materials on the dynamic seismic response of high slopes of highways, using the time histories of the 2008 Sichuan earthquake. The main conclusions are as follows: When the input seismic wave is a horizontal wave, the acceleration tended to increase with the elevation; there was a large displacement difference between slope top and slope foot; and the slope suffered tensile and shear failures under the action of the seismic wave. Bi (1979) summarized the law of the dynamic response of a slope to the Koyna seismic wave on Abaqus, revealing that the slope amplified the seismic acceleration; the horizontal peak acceleration at slope top was $1.0 \mathrm{~m} / \mathrm{s}^{2}$, about 3 times of the peak acceleration of the input seismic wave; and the vertical peak acceleration at the slope top was $0.8 \mathrm{~m} / \mathrm{s}^{2}$, about 3.2 times of the peak acceleration of the input seismic wave.

Chen and Chen (2004) explored the dynamic response law of slopes through repeated numerical simulations and summed up the distribution laws of the isolines of the acceleration, velocity, and displacement amplification factors on the profile of high rock slope under horizontal load: For a certain rock-soil mass, when the slope height is constant, the acceleration, velocity, and displacement, which are three quantities of the dynamic response of the slope, decrease with the growing slope angle; when the slope angle is constant, the acceleration, velocity, and displacement increase with the slope height; there is a certain regularity in the influence of the material properties of the underlying rock layer over the amplification of acceleration, velocity, and displacement; the response amplitude on the edge of the slope to the vibration is greater than that in the interior of the slope; the slope gradient determines the direction of the isolines of the three quantities. These conclusions clarify the features of dynamic seismic response of high rock slopes and theoretical and practical bases for high slope engineering. Nevertheless, the research on the dynamic seismic response of rock slopes is far from mature, owing to the complex geology of rock slopes, the stochasticity of seismic waves, and other uncertain factors (Liu et al. 2004).

When it comes to the mechanism and location of seismic instability of slopes, the influence of ground motion on slope stability is manifested as a cumulative effect and triggering effect (Luo et al. 2020; Nai 2010; Raza et al. 2020). The former refers to the plastic failure of rock-soil mass and the cumulative increase of pore water pressure of the slope, while the latter refers to that the ground motion induces the thixotropic softening of the weak layer, the liquefaction of the sand layer, and the instantaneous instability of the edge, which is already in the critical state.

Qi (2006) divided the seismic instability mechanisms of landslides into simultaneous mechanism and successive mechanism. The simultaneous mechanism involves the effects of both seismic inertial force and pore water pressure; the successive mechanism suggests that the arc-shaped seismic 
damage on the slope provides a channel for the penetration of groundwater, which can be considered the precursor of a landslide. Qi et al. (2004) categorized seismic slope instability as inertial instability and weakened instability. Rui and Zhang (1999) summarized the engineering geological features of seismic slope stability, pointing out that slope instability is the result of seismic inertial force, plus the rapid increase and accumulation of excess pore water pressure generated by the earthquake.

The type of landslide of a slope depends on the specific geological and tectonic structures of the slope. The landslides of different slopes are resulted from different factors. In general, the accumulation of pore water pressure plays a dominant role in plastic flow instability; the seismic inertial force is the decisive factor of slope collapse and layer bending; the cause of sliding failure varies with the specific conditions. Rather than an instantaneous overall failure, the sliding failure is a process as the local damage penetrates the slope profile, forming a slip surface (Sun 1999). So far, few numerical simulations have been carried out to clarify the mechanism of progressive failure and instability of slopes.

On the location of slope instability (usually the sliding surface) under seismic action, Tan (1998) classified the engineering geological models of slopes and the possible deformation and failure modes into two categories: the slopes with and without obvious controllable structural plane. The slopes in the first category are often rock slopes with weak interlayer, whose instability location can be determined through systematic engineering geological survey and monitoring. For the slopes in the second category, the instability location can be pinpointed by searching for the minimum safety factor in each calculation time step. The relevant search strategies include the Fibonacci method (Tang and Shao 2004; Wang 2013). The existing calculation results indicate that the location of the most dangerous sliding surface is relatively fixed (Yan 2011).

Miles and Ho (1999), the prerequisite for the overall instability of a slope is that the plastic zone of the sliding surface or the equivalent strain runs from the bottom to the top of the slope, but not all slopes of this type will form overall instability; second, when the soil mass on the sliding surface moves infinitely, it can be judged as the overall instability of the soil mass. At this time, the finite-element analysis results show that the strain or displacement on the sliding surface will change suddenly and the numerical calculation of force or displacement will not converge. The two methods in the courseware used approximately a same judgment basis. Therefore, we can use whether the force or displacement calculated by finite-element strength reduction is converge or not, or whether the plastic displacement and strain of each node on the rock mass sliding surface of the slope changes suddenly or not, as the basis for judging the overall failure of the slope.

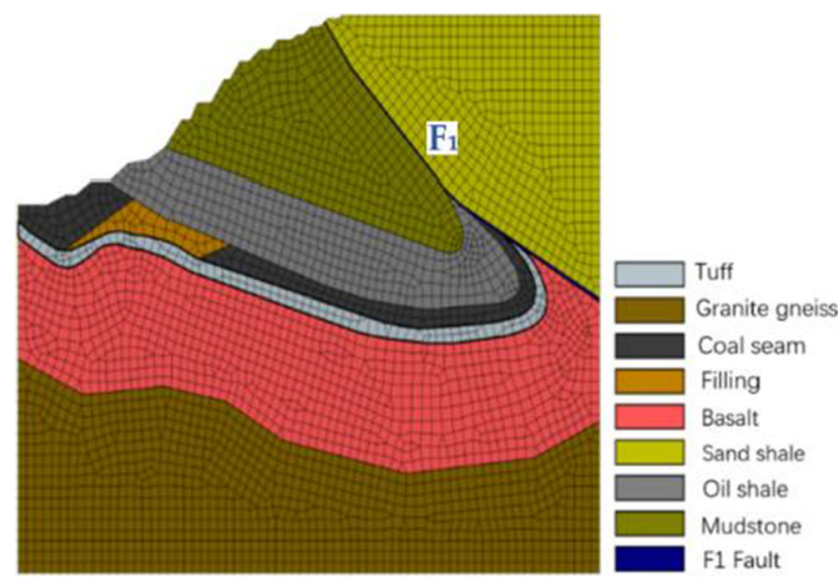

Fig. 1 The finite-element model of the northern slope of the West Pit mine

Penumadu and Zhao (1999) believe that the finite-element strength reduction applied an ideal elastoplastic model.

Through numerical simulation, this paper fully investigates the resonance features of the northern slope of the West Pit mine under seismic actions. The research findings reflect how rock slopes with anti-dip weak interlayer will resonant during the earthquake.

\section{Mechanical principles of slope resonance}

It is assumed that the target slope is a rigid body. The relevant research (Zang 1981) has shown that the slope will vibrate significantly, if its natural frequency is consistent with the frequency of the seismic wave. The obvious dynamic response of the slope is approximately a resonance.

In fact, a seismic wave contains multiple frequency components, while the slope, as a system with multiple degrees of freedom (DOFs), does not have a unique natural frequency (Zienkiewicz et al. 1975). Theoretically, it is possible for the natural frequency of the rigid slope to coincide with the frequency of the seismic wave, causing the slope to resonant with the seismic wave (Keefer 1984).

By finite-element analysis, the slope calculation model was treated as a multi-DOF system. The differential equation of its undamped free vibration can be expressed as

$M \ddot{x}+K x=0$

where $\mathrm{M}$ is the mass matrix; $\mathrm{K}$ is the stiffness matrix; and $\ddot{x}$ and $x$ are the column vectors of the acceleration and displacement of the system, respectively.

According to the general theory of differential equations, the basic solution of the undamped free vibration equation can be expressed as

$x_{(t)}=A \sin (\omega t+\phi)$ 


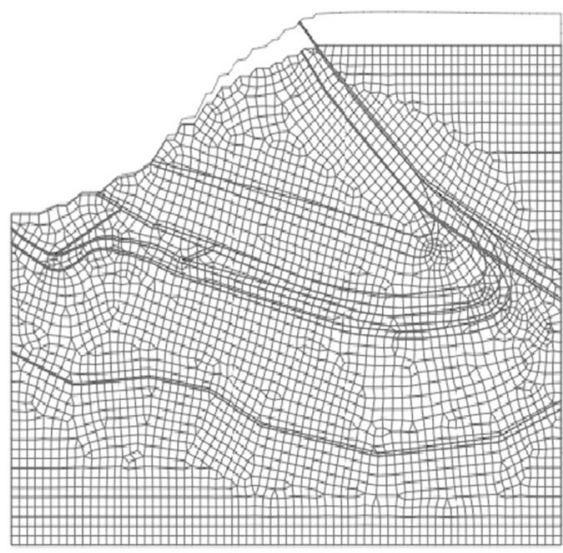

First-order vibration mode

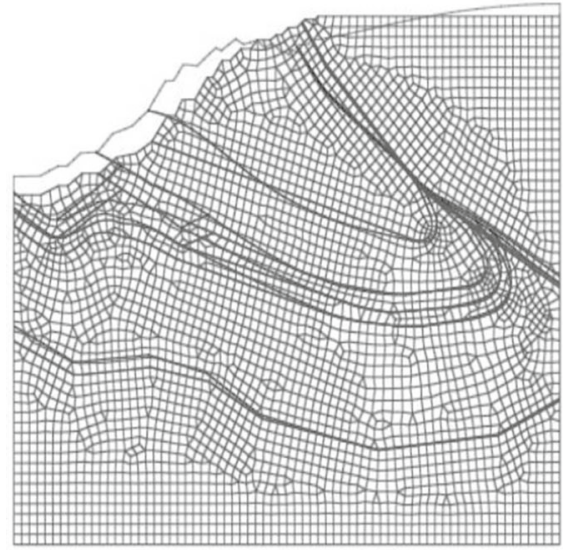

Fourth-order vibration mode

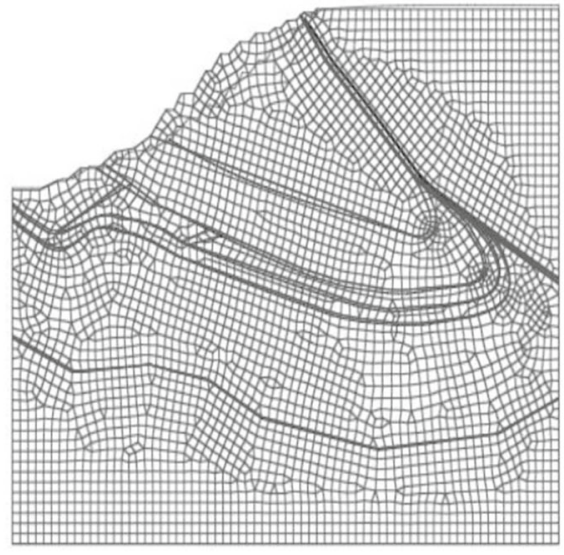

Second-order vibration mode

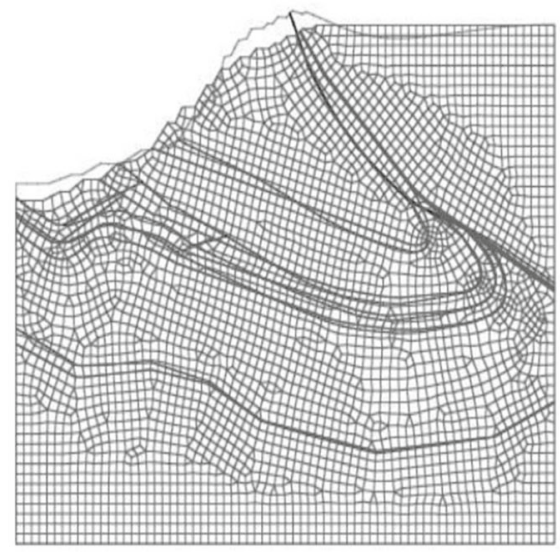

Fifth-order vibration mode

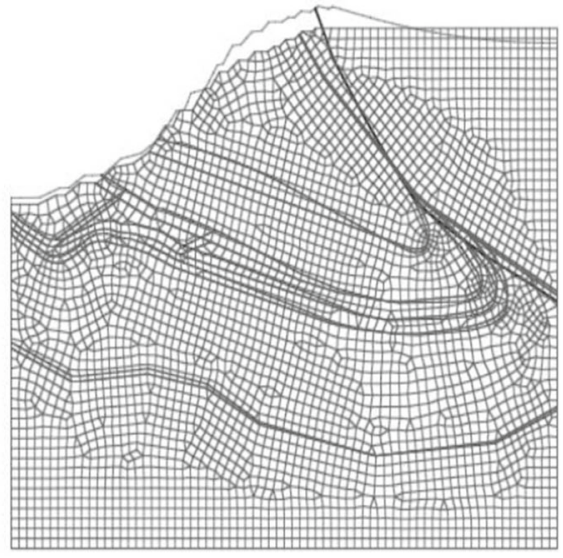

Third-order vibration mode

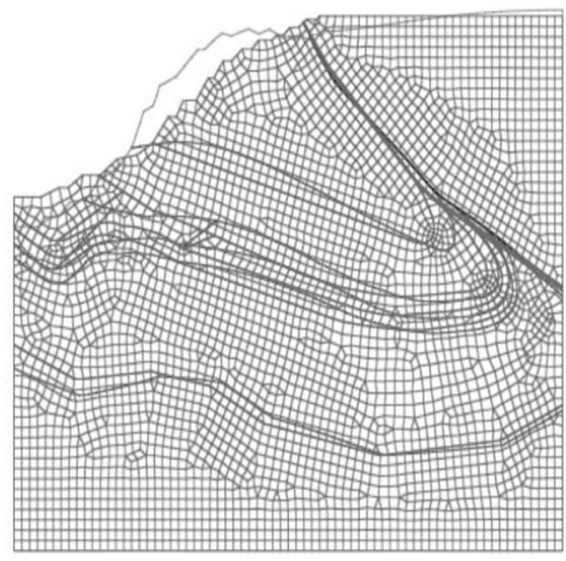

Sixth-order vibration mode

Fig. 2 The vibration modes of the first six orders of the slope

where $\mathrm{A}$ is an $\mathrm{n}$-dimensional non-zero column vector (the components are not related to time) and $\omega$ and $\phi$ are both $\quad\left(K-\omega^{2} M\right) A=0$ undetermined constants.

Table 1 The three modal extraction methods

\section{Method Application conditions}

Lanczo method Lanczo method is a powerful strategy applicable to most scenarios. It is very effective in extracting many modes $(40+)$ from medium to large models (with 50,000-100,000 DOFs), regardless of the presence of initial cut-off point. The method is often used in models with solid elements or shell elements. It can extract the vibration modes higher than a given frequency, and handle the vibration modes of rigid bodies well. However, a large memory is required by this method

Subspace $\quad$ Requiring a small memory, subspace method is suitable for extracting a few modes $(<40)$ from medium to large models. To apply this method method, the solid elements and shell elements should have good shapes, and the warning about element shape must be noted. This method might have a problem in convergence in extracting the vibration modes of rigid bodies. Do not use this method when there are constraint equations

Reduction The reduction method is faster than any other method, requiring a small memory and hard disk. It can be applied if the concentrated method mass (e.g., beams and rods) in the model does not induce local vibration. A set of DOFs is chosen to reduce the size of stiffness matrix $[\mathrm{K}]$ and mass matrix $[\mathrm{M}]$; the former is reduced accurately, but the latter is reduced approximately. The degree of approximation depends on the number and locations of the DOFs. If the structure (e.g., slender beams and thin shells) has a weak bending resistance, do not implement this method 
Table 2 The natural frequencies and periods of the slope

\begin{tabular}{ccc}
\hline Order & Natural frequency $(\mathrm{Hz})$ & Natural period (s) \\
\hline First & 1.138 & 0.917 \\
Second & 1.176 & 0.879 \\
Third & 1.380 & 0.850 \\
Fourth & 1.824 & 0.724 \\
Fifth & 2.044 & 0.548 \\
Sixth & 2.240 & 0.489 \\
\hline
\end{tabular}

According to the knowledge of linear algebra, the condition for the homogeneous linear equation of the components of A to have a non-zero solution is that the determinant of the coefficient matrix is zero. This condition can be expressed as a characteristic function:

$\operatorname{det}\left(K-\omega^{2} M\right)=0$

where $\omega_{i}^{2}$ is the eigenvalue. Let $f_{i}=\omega_{i} /(2 \pi)$ be the natural frequency of the system. The eigenvector $A_{i}$ corresponding to the eigenvalue $\omega_{i}^{2}$ is the vibration mode (Rodriguez et al. 1999).

\section{Numerical simulation of slope resonance}

\section{Modal analysis}

Modal analysis aims to determine the basic vibration features of the target structure. Through modal analysis, it is possible to obtain the natural frequency and vibration mode of the structure. The two parameters are essential to the design of a resonance-free structure and to dynamic analysis of various structures. Under different dynamic loads, the response of a structure depends on its vibration features. Hence, the vibration features of the structure must be fully understood to prevent the resonance. Therefore, modal analysis is the prerequisite for studies on the harmonic response, transient dynamics,

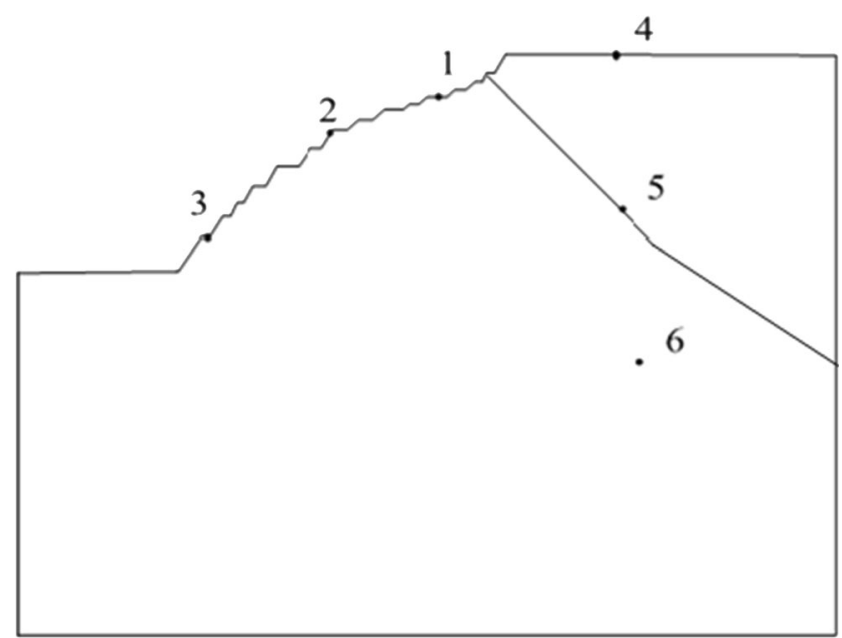

Fig. 3 The deployment of monitoring points

and spectrum of the target slope. The general form of dynamic analysis can be expressed as

$[M]\{\ddot{u}\}+[C]\{\dot{u}\}+[K]\{u\}=\{[F](t)\}$

In the case of free vibration and no damping:

$[M]\{\ddot{u}\}+[K]\{u\}=\{(0)\}$

If the vibration is harmonic motion:

$[K]-\omega^{2}[M]\{u\}=\{(0)\}$

where $\omega_{i}^{2}$ is the root of Formula (7) and the eigenvalue ( $\mathrm{i}$ is a number from 1 to the number of DOFs; the vector corresponding to $\omega_{i}^{2}$ is $\{u\}_{i}$, i.e., the eigenvector); $[M]$ and $[K]$ are constants (assuming that the structure is linear); $u=u_{0} \cos (\omega t)$ is the harmonic motion equation ( $\omega$ is the circular frequency of natural vibration, $\mathrm{rad} / \mathrm{s}$; the square of the eigenvalue is $\omega_{i}$, the natural circumference of the structure, $\mathrm{rad} / \mathrm{s}$; the natural frequency $\left.f_{i}=\omega_{i} / 2 \pi\right)$; and $\{u\}_{i}$ is the eigenvector representing the vibration mode, i.e., the structure shape vibrating at frequency $f_{i}$.

Table 3 The three harmonic response analysis methods

\begin{tabular}{ll}
\hline $\begin{array}{l}\text { Method } \\
\text { Reduction } \\
\text { method }\end{array}$ & $\begin{array}{r}\text { The law of the problem is compressed by using the main DOFs and reducing the matrix. After solving the displacements } \\
\text { corresponding to the main DOFs, the solutions can be extended to the initial complete set of DOFs. } \\
\text { The effect of preload is considered in this method. However, the loads must be applied to the user-defined DOFs, rather than the } \\
\text { elements }\end{array}$ \\
As the simplest method, the complete method calculates the harmonic response with the complete coefficient matrix, eliminating the \\
need for approximating the mass matrix or selecting main DOFs or vibration modes. The coefficient matrix can be symmetrical or \\
asymmetrical. All displacements and loads are computed through a single process. Various types of loads can be defined: nodal \\
force, applied non-zero displacement, and element load. In addition, the loads can be defined on the solid model. \\
There are two defects with this method: the preload option is not available, and the computing load is large sometimes \\
The structural response is calculated by multiplying the vibration modes, which are obtained through modal analysis, with factors, \\
and adding up the products. For many problems, this method has a smaller computing load than the previous two methods. \\
Both preload effect and damping can be considered in this method. But non-zero displacement is not allowed \\
Mode \\
superposition
\end{tabular}


Fig. 4 The horizontal displacement frequency responses of the surface monitoring points

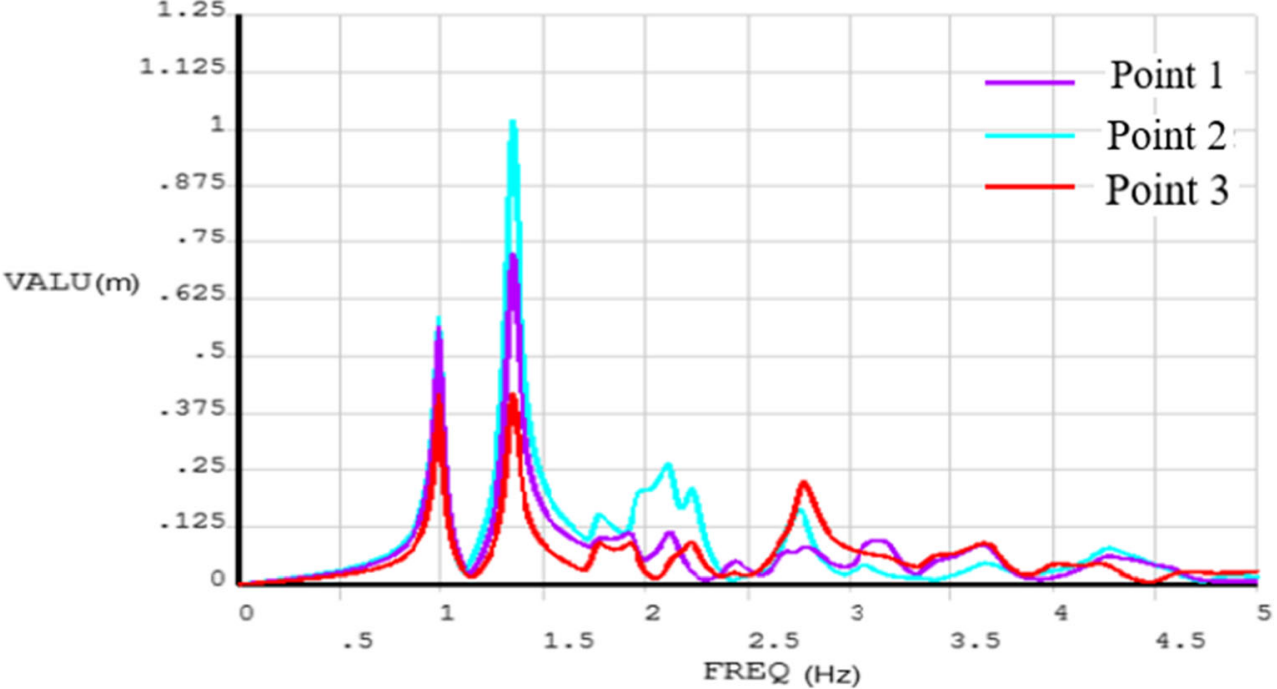

This paper uses midas GTS NX software for modal analysis. The Lanczo method was adopted to derive the natural frequencies and vibration modes of the target slope. Since the vibration features of the slope only hinge on low-order vibration modes, only the natural frequencies and periods in the first six orders are listed (Table 2) and illustrated (Figs. 1 and 2).

Table 1 briefly introduces three extraction methods for vibration modes.

The vibration modes in the first six orders of the slope were displayed in deformed and undeformed grids. As shown in Figs. 1 and 2, the first-order vibration mode is vertical vibration, the second-order vibration mode is horizontal vibration, the third- and fifth-order vibration modes are high-order vibrations dominated by vertical vibration, and the fourth- and sixth-order vibration modes are high-order vibrations dominated by horizontal vibration. From these vibration modes, it can be inferred that the vibration of the slope mainly takes place on the surface; that is, the surface vibration is relatively strong during the earthquake. The surface is also a weak part of the slope. Therefore, the slope damage is very likely to begin from the slope. Seismic waves of different frequencies will excite different vibration modes. Resonance will occur once the frequency of the seismic wave coincides with a certain natural frequency of the slope.

\section{Harmonic response analysis}

Harmonic response refers to the continuous periodic response of the structure to continuous periodic loads. Harmonic response analysis can generally determine the steady-state response of linear structures under simple harmonic loads, and output a series of structural responses can be obtained at different frequencies. The correlations between these responses and the corresponding frequencies can be illustrated by harmonic response-frequency curves. Then, the harmful effects
Fig. 5 The vertical displacement frequency responses of the surface monitoring points

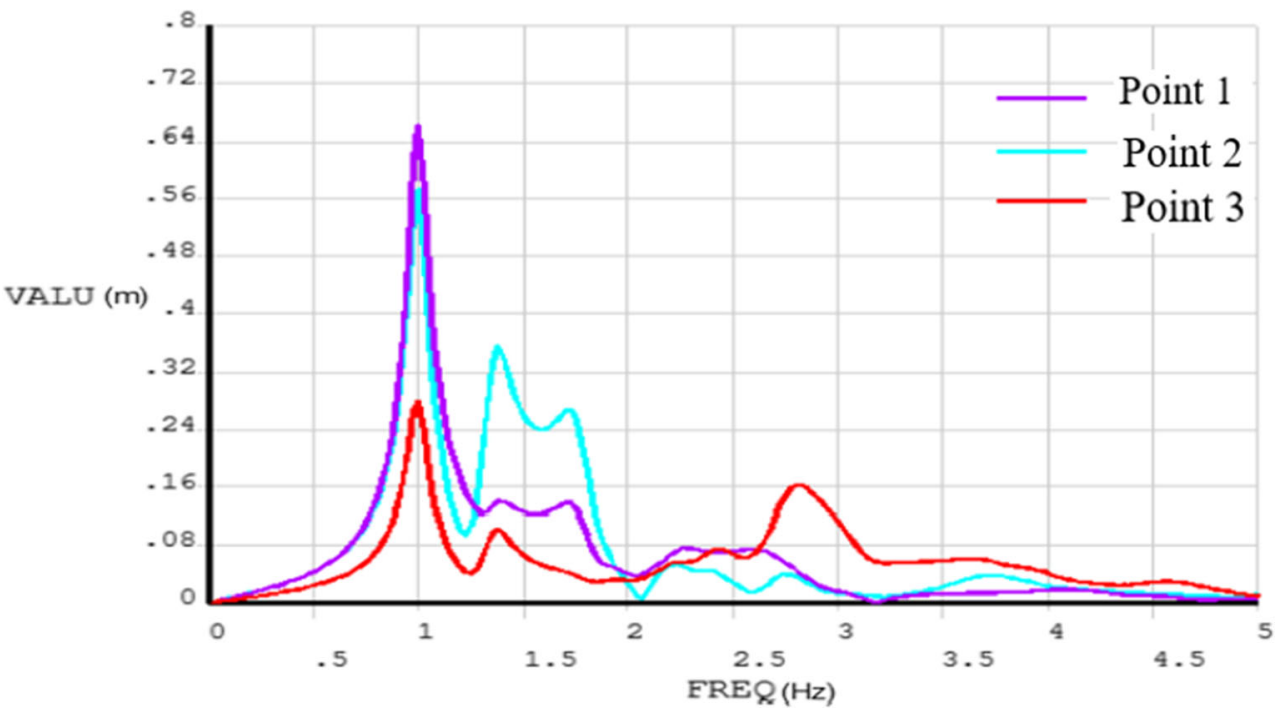


Fig. 6 The horizontal displacement frequency responses of the interior monitoring points

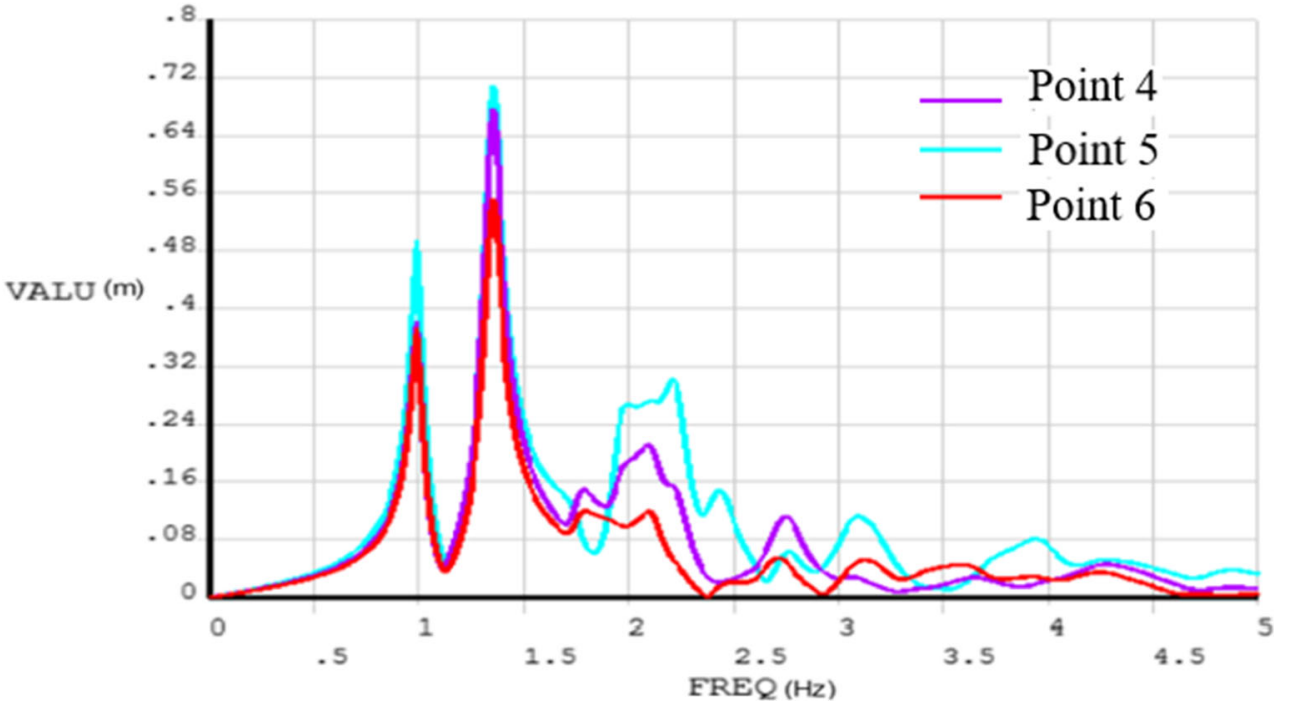

of resonance on the slope can be predicted based on the peak responses and the corresponding displacements on the curves.

Without considering nonlinear features, harmonic response analysis helps to disclose the continuous dynamic features of the target structure and reveal the adverse dynamic structural responses (e.g., resonance and fatigue) under forced vibration so that these adverse responses can be solved in structural design. The three popular methods of harmonic response analysis are introduced in Tables 2 and 3.

The general form of the dynamic analysis equation can be expressed as

$[M]\{\ddot{u}\}+[C]\{\dot{u}\}+[K]\{u\}=\{F\}$

where $\{\mathrm{F}\}$ and $\{\mathrm{u}\}$ are harmonic matrices at the frequency of $\omega:$

$\{F\}=\left\{F_{\max } e^{i \psi}\right\} e^{i \omega t}=\left(\left\{F_{1}\right\}+i\left\{F_{2}\right\}\right) e^{i \omega t}$ $\{u\}=\left\{u_{\max } e^{i \psi}\right\} e^{i \omega t}=\left(\left\{u_{1}\right\}+i\left\{u_{2}\right\}\right) e^{i \omega t}$

The dynamic equation for harmonic response analysis can be expressed as

$$
\begin{aligned}
& \left(-\omega^{2}[M]+i \omega[C]+[K]\right)\left(\left\{u_{1}\right\}+i\left\{u_{2}\right\}\right. \\
& \quad=\left(\left\{F_{1}\right\}+i\left\{F_{2}\right\}\right)
\end{aligned}
$$

where $F_{\max }$ is the load amplitude; $\psi$ is the phase angle of the load function; $F_{1}=F_{\max } \cos \psi$ and $F_{1}=F_{\max } \sin \psi$ are the real and imaginary parts of the phase angle, respectively; $u_{\max }$ is the displacement amplitude; and $u_{1}=u_{\max } \cos \varphi$ and $u_{2}=$ $u_{\max } \sin \varphi$ are the real and imaginary parts of the displacement amplitude, respectively.

The complete method was selected for model calculation. To fully reflect the slope response at different positions, monitoring points were deployed on the surface and within the slope (Fig. 3).
Fig. 7 The vertical displacement frequency responses of the interior monitoring points

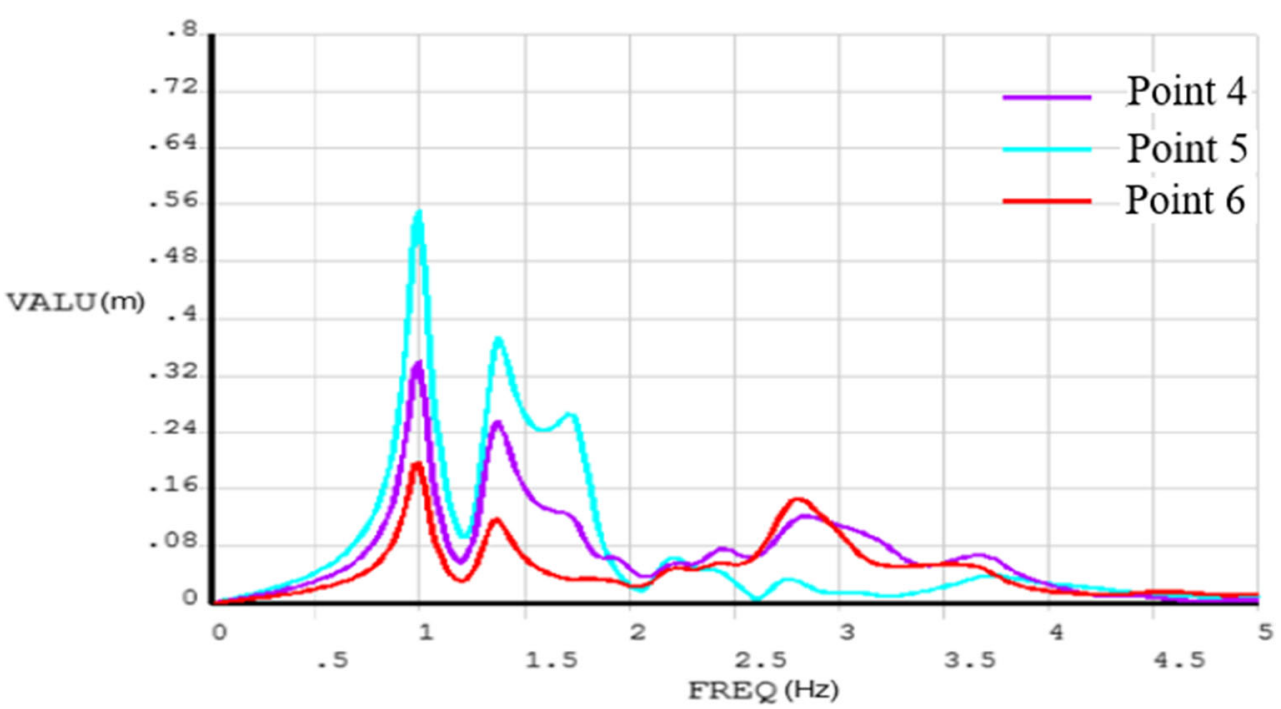




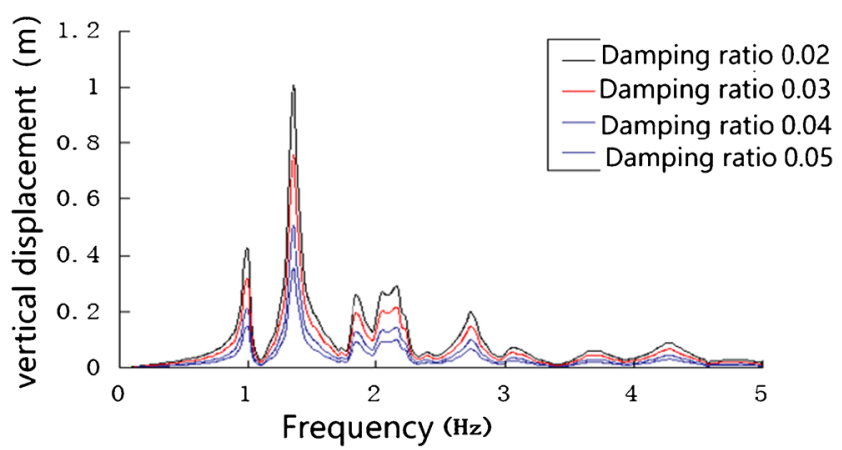

Fig. 8 The vertical displacement frequency curves of point 4

Based on modal analysis, a simple harmonic wave with an amplitude of $0.2 \mathrm{~g}$ was input at the bottom of the model for excitation. With a damping ratio of 0.02 , the wave was loaded horizontally at the frequency of $0-5 \mathrm{~Hz}$. Then, the frequency response curve of each point was plotted based on the results of harmonic response analysis (Figs. 4, 5, 6, and 7).

As shown in Figs. 5 and 7, the vertical resonance peak of the monitoring points appeared at the same frequency at the frequency of about $1.1 \mathrm{~Hz}$. This resonance frequency is roughly the same as the first-order natural frequency $(1.138 \mathrm{~Hz})$. Two monitoring points had obvious resonance peaks in horizontal displacement, which appeared at around the second-, and third-order natural frequencies $(1.17$ and $1.80 \mathrm{~Hz})$, respectively.

As shown in Figs. 4 and 6, the slope resonance is mainly controlled by the vibration modes of the first three orders. The displacement response curves of point 5 indicate that the large damping of the rock-soil mass with poor integrity can weaken the seismic impact to a certain extent; but the broken structure makes the mass prone to damage.

Taking point 4 as the target, the resonance response curves of the slope were calculated at the damping ratios of 0.02 , $0.03,0.04$, and 0.05 , respectively. Figure 8 presents the vertical displacement frequency curves of point 4 at different damping ratios. It can be seen that the resonance peak, i.e. vertical displacement, is negatively correlated with the damping ratio.

\section{Conclusions}

This paper analyzes the resonance features of the northern slope of West Pit mine. Firstly, the natural frequencies and vibration modes of the slope were obtained through modal analysis. Then, the harmonic response analysis was performed to draw the resonance curves of the slope, revealing the relationship between displacement and frequency. It is concluded that the resonance effect of the slope is mainly excited by the natural frequencies of the first three orders: the vertical resonance displacement peaked at about $1.1 \mathrm{~Hz}$, while the horizontal resonance displacement peaked at about $1.38 \mathrm{~Hz}$. From the vibration modes and frequency response curves, the resonance peak of the slope is amplified in the vertical direction and on the free face, and the resonance displacement is greater on the surface than in the interior of the slope; i.e., the resonance is more prominent on the surface than in the interior. Moreover, the resonance response law of the slope was calculated under different damping ratios. Comparing the frequency response curves at different monitoring points, it is learned that slope resonance is greatly affected by damping ratio: the greater the damping ratio, the lower the resonance peak; the inverse is also true. This means that weak and broken rock mass is capable of absorbing shocks, but not necessarily easy to be damaged. On the contrary, broken rock mass has poor mechanical performance, and is prone to damage under small vibration.

Funding This work was supported by Science and Technology Innovation Fund for Small and Medium-Sized Enterprises (Grant No. 320180025), and Department of Education, Jilin Province, China (Grant No. JJKH20191268KJ).

Open Access This article is licensed under a Creative Commons Attribution 4.0 International License, which permits use, sharing, adaptation, distribution and reproduction in any medium or format, as long as you give appropriate credit to the original author(s) and the source, provide a link to the Creative Commons licence, and indicate if changes were made. The images or other third party material in this article are included in the article's Creative Commons licence, unless indicated otherwise in a credit line to the material. If material is not included in the article's Creative Commons licence and your intended use is not permitted by statutory regulation or exceeds the permitted use, you will need to obtain permission directly from the copyright holder. To view a copy of this licence, visit http://creativecommons.org/licenses/by/4.0/.

\section{References}

Bi ZW (1979) Discussion on the relationship between earthquake and landslide and earthquake landslide prediction. People's Railway Press, Bei Jing, pp 127-132

Cardarilli M, Lombardi M, Corazza A (2019) Landslide risk management through spatial analysis and stochastic prediction for territorial resilience evaluation. Int J Saf Sec Eng 9(2):109-120

Chen LL, Chen MZ (2004) Graduate School of Chinese Academy of Sciences. Grad School Chin Acad Sci 25(11):11786-11790

Du JC, Huang DS, Hu D (2001) Three-dimensional method of limiting equilibrium for stability analysis of slope. J Sichuan Univ Eng Sci Ed 33(4):9-12

Gao CJ, Qiao D, Zhang XF, Liu SY, Zhou YF (2019) Analysis of seepage influence on high slopes based on multi-dimensional measured data. Instrum Mesure Métrol 18(6):603-613

Keefer DK (1984) Landslides caused by earthquakes. Geol Soc Am Bull 95(4):406-421

Li C, Cai Y (2020) Effect of soil strength degradation on slope stability. Int J Des Nat Ecodyn 15(4):483-489

Liu HS, Bo JS, Geng DQ, Wu ZY (2004) Rock landslide stability analysis by finite element method. Rock Soil Mech 25:1786-1790

Luo JH, Mi DC, Huang HF, Zhang T, Sun GH, Chen DQ (2020) Intelligent monitoring, stability evaluation, and landslide treatment 
of a carbonaceous mudstone and shale slope in Guangxi, China. Int J Saf Sec Eng 10(3):373-379

Miles SB, Ho CL (1999) Rigorous landslide hazard zonation using Newmark's method and stochastic ground motion simulation. Soil Dyn Earthq Eng 18(4):305-323

Nai L (2010) Slope Engineering. The Science Publishing Company, Bei Jing

Onyango JA, Zhang CY (2019) Numerical analysis of slope stability by strength reduction in finite elements using ANSYS a case study of Qinglong-Xingyi expressway contract section T1(K11 + 790 K11 + 875). Environ Earth Sci Res J 6(2):89-96

Penumadu D, Zhao R (1999) Triaxial compression behavior of sand and gravel using artificial neural networks (ANN). Comput Geotech 24(3):207-230

Qi SW (2006) Analysis and application of slope dynamic response. Graduate School of Chinese Academy of Sciences, Bei Jing

Qi SW, Wu FQ, Liu CL, Ding YH (2004) Engineering geology analysis on stability of slope under earthquake. Chin J Rock Mech Eng 23(16):2792-2797

Qiu CJ, Wang S, Liu H, Huang J (2018) In-situ pull-out tests on soilreinforcement interface properties of reinforced soil slopes. Ann Chim Sci Mater 42(1):41-56

Raza M, Khan F, Khan MY, Riaz MT, Khan U (2020) Reservoir characterization of the B-interval of lower goru formation, miano 9 and 10, miano area, Lower Indus Basin, Pakistan. Environ Earth Sci Res J 7(1):18-32

Rodriguez CE, Bommer JJ, Chandler RJ (1999) Earthquake-induced landslides: 1980-1997. Soil Dyn Earthq Eng 18(5):325-346

Rui YQ, Zhang YD (1999) Dynamic control technology of creep slope deformation and failure in open pit coal mine. China Mining 8(4): 34-38

Shuren FENG, Dingxiang F, Xiurun G, Xianrong G (1999) 3D limit equilibrium method for slope stability and its application. Chin J Geotech Eng-Chin Ed 21(6):657-661
Sun YK (1999) Study on slope stability of open pit mines in China. Press of University of Science and Technology of China, Bei Jing

Tan WH (1998) Discussion on slope stability analysis method. Open Pit Min Technol 2:21-23

Tang HX, Shao LT (2004) Finite Element Analysis on Slope Stability of Earth-Rock Dam Under Earthquake. Chin J Rock Mech Eng 23(8): $1318-1324$

Wang LG (2013) System analysis of environmental disaster in resource exhausted coal city. Geological Publishing House, Bei Jing

Xu GX (2006) Earthquake engineering. Seismological Press, Bei Jing

Yan ZX (2011) Study on resonance characteristics and natural frequency of slope under bidirectional seismic action. Hydrogeol Eng Geol $38(2): 46-51$

Yang J (2007) The Long-term stability reliability of the earth slope by seismic action. Fujian ConstructSci Technol 2:8-9,19

Zang ZY (1981) Principles of engineering geological analysis. Geological Publishing House, Bei Jing

Zhang DQ (1998) Analysis on mechanism and monitoring data of slope wetting and sliding in a project. Chin J Rock Mech Eng 17(2):133139

Zhang H (2008) Mechanism and geomechanical modes of landslide hazards triggered by Wenchuan 8.0 earthquake. Chin J Rock Mech Eng 28(6):1239-1249

Zheng LM (2000) Vibration characteristics and mechanism of rock mass in railway slope. Chin J Geotech Eng 13(1):69-78

Zhou DP (2010) Analysis on earthquake damage of road slope engineering in Wenchuan County earthquake. Chin J Geotech Eng 29(3): $565-576$

Zienkiewicz OC, Humpheson C, Lewis RW (1975) Associated and nonassociated visco-plasticity and plasticity in soil mechanics. Geotechnique 25(4):671-689 